\title{
Comparing the mitochondrial genomes of Wolbachia-dependent and independent filarial nematode species
}

\author{
Samantha N McNulty ${ }^{1 *}$, Andrew S Mullin', Jefferson A Vaughan², Vasyl V Tkach², Gary J Weil ${ }^{1}$ and Peter U Fischer ${ }^{1}$
}

\begin{abstract}
Background: Many species of filarial nematodes depend on Wolbachia endobacteria to carry out their life cycle. Other species are naturally Wolbachia-free. The biological mechanisms underpinning Wolbachia-dependence and independence in filarial nematodes are not known. Previous studies have indicated that Wolbachia have an impact on mitochondrial gene expression, which may suggest a role in energy metabolism. If Wolbachia can supplement host energy metabolism, reduced mitochondrial function in infected filarial species may account for Wolbachia-dependence. Wolbachia also have a strong influence on mitochondrial evolution due to vertical co-transmission. This could drive alterations in mitochondrial genome sequence in infected species. Comparisons between the mitochondrial genome sequences of Wolbachia-dependent and independent filarial worms may reveal differences indicative of altered mitochondrial function.

Results: The mitochondrial genomes of 5 species of filarial nematodes, Acanthocheilonema viteae, Chandlerella quiscali, Loa loa, Onchocerca flexuosa, and Wuchereria bancrofti, were sequenced, annotated and compared with available mitochondrial genome sequences from Brugia malayi, Dirofilaria immitis, Onchocerca volvulus and Setaria digitata. B. malayi, D. immitis, O. volvulus and W. bancrofti are Wolbachia-dependent while A. viteae, C. quiscali, L. loa, O. flexuosa and S. digitata are Wolbachia-free. The 9 mitochondrial genomes were similar in size and AT content and encoded the same 12 protein-coding genes, 22 tRNAs and 2 rRNAs. Synteny was perfectly preserved in all species except C. quiscali, which had a different order for 5 tRNA genes. Protein-coding genes were expressed at the RNA level in all examined species. In phylogenetic trees based on mitochondrial protein-coding sequences, species did not cluster according to Wolbachia dependence.

Conclusions: Thus far, no discernable differences were detected between the mitochondrial genome sequences of Wolbachia-dependent and independent species. Additional research will be needed to determine whether mitochondria from Wolbachia-dependent filarial species show reduced function in comparison to the mitochondria of Wolbachia-independent species despite their sequence-level similarities.
\end{abstract}

\section{Background}

Filarial nematodes are arthropod borne parasitic worms that infect hundreds of millions of people throughout the tropics and sub-tropics and are responsible for a great deal of morbidity in humans and domestic animals. Many filarial pathogens, such as the agents of lymphatic filariasis and river blindness, require a bacterial

\footnotetext{
* Correspondence: smcnulty@wustl.edu

${ }^{1}$ Infectious Diseases Division, Department of Internal Medicine, Washington University School of Medicine, Campus Box 8051, 660 S. Euclid Avenue, St. Louis, MO 63110, USA

Full list of author information is available at the end of the article
}

endosymbiont, Wolbachia pipientis, to carry out their life cycle [1-4]. In these species, depletion of the endosymbiont causes defects in growth, molting and fertility, leading to the death of the worm [5-7]. Other filarial species, some of which are very closely related to Wolbachia-dependent sister taxa, are naturally Wolbachia-free [1,2,8-10]. Thus far, there are no discernable patterns in Wolbachia distribution (e.g., based on host species, vector species, tissue tropism, geographic distribution, etc.), and the reasons for this disparity are poorly understood. Presumably, some genetic function(s) must be missing or reduced in Wolbachia-dependent worms in comparison to their
C Biomed Central

(c) 2012 McNulty et al.; licensee BioMed Central Ltd. This is an Open Access article distributed under the terms of the Creative Commons Attribution License (http://creativecommons.org/licenses/by/2.0), which permits unrestricted use, distribution, and reproduction in any medium, provided the original work is properly cited. 
Wolbachia-free counterparts, forcing them to rely on the bacteria as an alternative source of vital gene products. The processes underpinning Wolbachia-dependence are of biological and medical interest, as the Wolbachia products required by the dependent worm may represent useful targets for novel anti-filarial chemotherapies.

Wolbachia endobacteria and the eukaryotic mitochondria share many common features, including the intracellular lifestyle, obligatory mutualism, reduced genome size, vertical transmission, etc. These shared features, as well as their shared ancestry in the order Rickettsiales [11-13], lead us to hypothesize that Wolbachia may contribute to energy metabolism in the filarial host. Previous studies have shown that antibiotic-mediated Wolbachia depletion leads to upregulation in genes related to energy metabolism, including mitochondrially encoded subunits of the respiratory chain [14]. This impact on host mitochondrial gene expression, and presumably energy production, suggests that Wolbachia may serve as an alternative energy source or mitochondrial "supplement," necessitating increased activity when the endosymbiont is removed. If so, differences in mitochondrial function may account for discrepancies in Wolbachia status in the filarial lineage.

The mitochondrial genome (mtDNA) is particularly sensitive to evolutionary pressure exerted by the Wolbachia infection. Vertically-transmitted Wolbachia are able to expand rapidly through insect populations due to the mechanisms of reproductive parasitism [15]. Wolbachia and mitochondria are co-transmitted. Thus, the mtDNA(s) of the first infected individual(s) presumably expand concurrently with the Wolbachia infection. Such Wolbachia-mitochondria "sweeps," characterized by unusually low degrees of variation in the mtDNA of infected populations, have been noted in many insect species [16-20]. A similar lack of mtDNA diversity is seen in populations of Dirofilaria immitis (canine heartworm) in comparison to Wolbachia-free, non-filarial nematodes [21]. A Wolbachia-induced genetic bottleneck may have led to the fixation of different mtDNA types among infected filarial species as compared to uninfected species.

The mtDNA sequences of 4 species of filarial nematodes, Onchocerca volvulus [22], D. immitis [23], Brugia malayi [24], and Setaria digitata [25], have been published. This report details the sequencing and analysis of the mtDNA sequences of 5 more species: Acanthocheilonema viteae, Chandlerella quiscali, Loa loa, Onchocerca flexuosa and Wuchereria bancrofti. Studies of the distribution of Wolbachia within filarial nematodes have shown that the infection is prevalent among 2 of the 8 filarial subfamiles, the Onchocercinae and the Dirofilariinae [1,2]. Agreement between the phylogenies of Wolbachia and their filarial hosts suggests that Wolbachia entered the filarial lineage prior to the diversification of these 2 subfamilies [1,26]. 7 of the 9 species included in this study are members of the the Onchocercinae and Dirofilariinae. Four of these, B. malayi, D. immitis, O. volvulus and W. bancrofti, are Wolbachiadependent $[1,3,4,27]$. The other 3, A. viteae, L. loa and O. flexuosa, are Wolbachia-free $[1,8,10,28]$, presumably due to secondary loss of the endosymbiont $[1,29]$. Conversely, $C$. quiscali and $S$. digitata are Wolbachia-free and belong to subfamilies (Splendidofilariinae and Setariinae, respectively) that have not been shown to contain Wolbachia-infected species, suggesting that these subfamilies split from the lineage prior to the introduction of Wolbachia endobacteria [2,9].

In light of the presumed impact of Wolbachia on the host mitochondria, we hypothesized that the mtDNAs of Wolbachia-dependent filaria may differ in gene content, arrangement or sequence as compared to those found in Wolbachia-free species whose ancestor(s) may not have undergone a Wolbachia-induced genetic bottleneck or evolved in the presence of an endobacterial partner capable of affecting host energy metabolism. The purpose of the reported study was to compare mtDNA from Wolbachiadependent and independent filarial species in search of sequence level differences indicative of altered mitochondrial function. Our analyses revealed no differences that could be attributed to Wolbachia status. Future studies will be required to discover subtler affects of Wolbachia on the sequence or function of filarial nematode mitochondria.

\section{Results}

\section{Gene content and organization}

The mtDNAs of 5 species of filarial nematodes were sequenced, annotated and deposited in Genbank (see Table 1 for accession numbers). Genome length, ATrichness and base composition of the $9 \mathrm{mtDNAs}$ are compared in Table 1 . The newly sequenced mtDNAs are similar in size and AT content to those of other filarial species. So far, filarial mtDNAs range in size from 13,474 bp in O. volvulus to 13,839 in S. digitata and range in AT content from $73.7 \%$ in O. volvulus to $77.7 \%$ in C. quiscali [22,25].

All 9 filarial mtDNAs encode the same 12 proteins, 22 tRNAs and 2 rRNAs with very short intergenic sequences (Figure 1). These genes are encoded in the same direction, a characteristic shared by most nematode mtDNAs. Synteny is perfectly preserved in all examined species with the exception of $C$. quiscali (Figure 1). In 8 of the 9 species, 5 tRNA genes (tRNA ${ }^{\text {Ala }}$, tRNA ${ }^{\text {Leu2 }}$, $\mathrm{tRNA}^{\mathrm{Asn}}$, $\mathrm{tRNA}^{\text {Met }}$ and $\mathrm{tRNA}^{\text {Lys }}$ ) reside between the ATrich region and NDL4. In C. quiscali, the tRNA ${ }^{\text {Met }}$ gene is positioned between Cox3 and the AT-rich region apart from the main tRNA cluster, and the order of the other 4 tRNA genes is rearranged relative to other species. For a comparisons between the mtDNA arrangement among filarial and other nematodes, see [25]. 
Table 1 Comparison of filarial nematode mtDNAs

\begin{tabular}{|c|c|c|c|c|c|c|c|c|c|}
\hline \multirow[b]{2}{*}{ Species } & \multicolumn{4}{|c|}{ Wolbachia-dependent species } & \multicolumn{5}{|c|}{ Wolbachia-independent species } \\
\hline & B. malayi & D. immitis & O. vovlulus & W. bancrofti & A. viteae & L. loa & O. flexuosa & C. quiscali & S. digitata \\
\hline Subfamily & Onchocercinae & Dirofilariinae & Onchocercinae & Onchocercinae & Onchocercinae & Dirofilariinae & Onchocercinae & Splendidofilariinae & Setariinae \\
\hline Accession Number & NC_004298 & NC_005305 & NC_001861 & HQ184469 & HQ186249 & HQ186250 & HQ214004 & HM773029 & NC_014282 \\
\hline Length (bp) & 13,657 & 13,814 & 13,474 & 13,636 & 13,724 & 13,590 & 13,672 & 13,757 & 13,839 \\
\hline $\begin{array}{l}\text { Length of AT-rich } \\
\text { region (bp) }\end{array}$ & 283 & 362 & 312 & 256 & 421 & 288 & 284 & 308 & 506 \\
\hline$A \%$ & $21.60 \%$ & $19.30 \%$ & $19.30 \%$ & $20.50 \%$ & $19.60 \%$ & $20.80 \%$ & $20.30 \%$ & $23.00 \%$ & $19.40 \%$ \\
\hline $\mathrm{T} \%$ & $53.90 \%$ & $54.90 \%$ & $54.00 \%$ & $54.10 \%$ & $54.00 \%$ & $54.80 \%$ & $53.90 \%$ & $54.70 \%$ & $55.70 \%$ \\
\hline $\mathrm{G} \%$ & $16.80 \%$ & $19.30 \%$ & $19.80 \%$ & $18.00 \%$ & $19.30 \%$ & $17.70 \%$ & $18.60 \%$ & $15.90 \%$ & $18.20 \%$ \\
\hline $\mathrm{C} \%$ & $7.70 \%$ & $6.50 \%$ & $6.90 \%$ & $7.40 \%$ & $7.20 \%$ & $6.70 \%$ & $7.20 \%$ & $6.40 \%$ & $6.70 \%$ \\
\hline AT\% & $75.50 \%$ & $74.20 \%$ & $73.30 \%$ & $74.60 \%$ & $73.50 \%$ & $75.60 \%$ & $74.20 \%$ & $77.70 \%$ & $75.10 \%$ \\
\hline
\end{tabular}

Information was taken from previous studies for B. malayi [24], D. immitis [23], O. volvulus [22], and S. digitata [25]. Information regarding the Wolbachia status of species from various subfamilies is reported in [2]. 


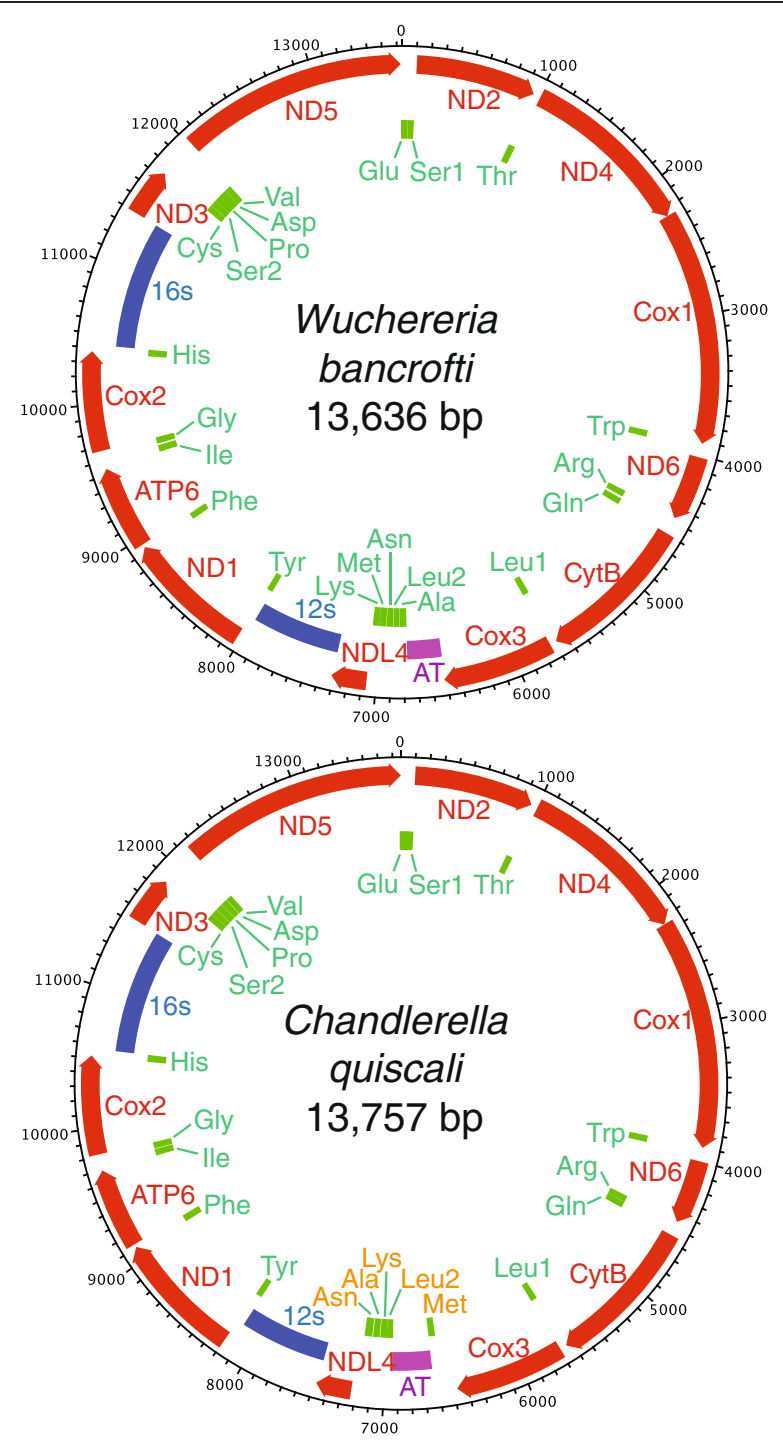

Figure 1 Comparative diagrams of the mitochondrial genomes of $\boldsymbol{W}$. bancrofti and $\boldsymbol{C}$. quiscali. Protein-coding genes are shown in red with arrowheads indicating directionality. rRNA and tRNA genes are shown in blue and green, respectively, and the AT-rich region is shown in purple. The diagram of the $W$. bancrofti mitochondrial genome is representative of most filarial mitochondria, as synteny is preserved in all species except C. quiscali. The 5 tRNA genes rearranged in C. quiscali are highlighted in orange.

\section{Protein-coding genes}

Twelve protein-coding genes were identified in each of the examined mtDNAs. None of these contain premature stop codons or frameshift mutations. Reverse transcription PCR reactions indicate that the predicted protein-coding genes were expressed at the RNA level in all examined species (Figure 2).

Filarial mtDNAs are extremely thymine (T)-rich (Table 1); therefore, it is not surprising that filarial mitochondria show a bias towards T-rich codons (Additional file 1: Table S1). The most frequently used codon in all species is TTT, which encodes phenylalanine and serves as an alternative start codon in certain instances (Additional file 1: Table S1, Table 2). The start and stop codons used by each species are listed in Table 2. Novel start codons include TGT for ND6 in $W$. bancrofti, TCT for CytB in A. viteae, and CCT for ND3 in O. flexuosa. Termination codons include TAG, TAA, and the incomplete stop codon $\mathrm{T}$, which is converted to TAA upon addition 3' poly(A) tail.

\section{Ribosomal and transfer RNA genes}

All species encode 2 rRNA genes. In all species examined, the 12s rRNA gene is positioned between NDL4 and ND1 while the 16s rRNA gene is positioned between Cox 2 and ND3 (Figure 1). The exact boundaries of these genes have yet to be mapped in any filarial species.

All species also contain the same 22 tRNA genes. In the previously sequenced species, 20 of the 22 mitochondrial 


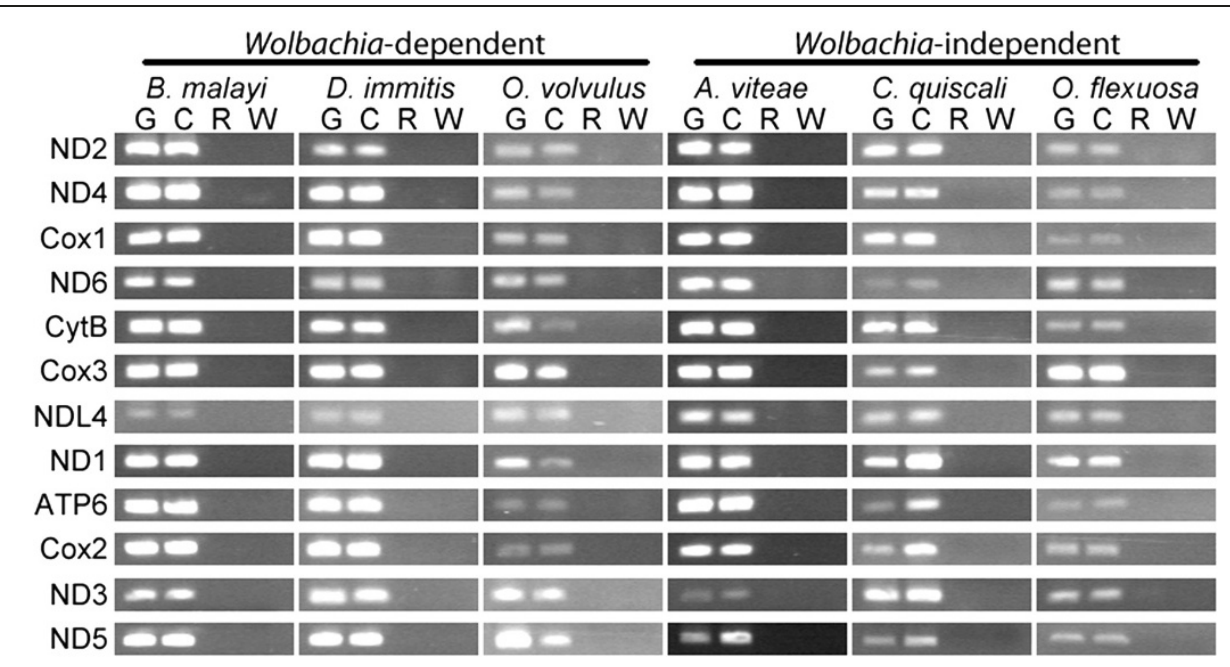

Figure 2 Expression of mitochondrial protein-coding genes in six filarial nematode species. Expression of mitochondrial protein-coding genes was assessed by reverse transcription PCR in B. malayi, D. immitis, O. volvulus, A. viteae, C. quiscali and O. flexuosa. The following templates were used for each reaction: genomic DNA (G), CDNA (C), total RNA (R) and water (W).

tRNAs share a common secondary structure in which the TYC arm and variable loop are exchanged for a TVreplacement loop [22,23] (Figure 3b). Conversely, the two tRNA ${ }^{\text {Ser }}$ genes contain a DHU replacement loop in exchange for the typical D arm (Figure 3c) [22,23]. The predicted mitochondrial tRNA structures of $A$. viteae followed this trend exactly, as did most of the tRNAs from the other examined species. However, our predictions indicate that tRNA ${ }^{\text {Ser1 }}$ and tRNA ${ }^{\text {Asn }}$ in C. quiscali, tRNA $^{\text {Lys }}$ and tRNA ${ }^{\text {Pro }}$ in $L$. loa, tRNA $^{\text {Trp }}$ in O. flexuosa and tRNA ${ }^{\text {Pro }}$ in $W$. bancrofti may contain both the TYC and D loops (Figure 3d-i). The same anticodons are used in all species with two exceptions. tRNA ${ }^{\text {Pro }}$ uses the anticodon AGG in O. volvulus, D. immitis, S. digitata and $O$. flexuosa, while the anticodon TGG is used in other species, and tRNA ${ }^{\mathrm{Leu} 1}$ uses the anticodon TAA in $A$. viteae while the anticodon TAG is used in other species.

\section{AT rich region}

The control, or AT rich, region represents the largest noncoding region in filarial mtDNAs, which are otherwise densely packed with tightly spaced or slightly overlapping

Table 2 Start and stop codons used in mitochondrial protein-coding genes

\begin{tabular}{|c|c|c|c|c|c|c|c|c|c|}
\hline & \multicolumn{4}{|c|}{ Wolbachia-dependent species } & \multicolumn{5}{|c|}{ Wolbachia-independent species } \\
\hline & B. malayi & D. immitis & O. volvulus & W. bancrofti & A. viteae & L. $10 a$ & O. flexuosa & C. quiscali & S. digitata \\
\hline$\overline{N D 2}$ & TTA/T & ATT/TAG & ATT/TAG & TTA/T & TT/TAG & ATT/TAA & ATT/TAG & ATT/TAG & $\pi T / T A G$ \\
\hline ND4 & TTG/TAA & TTG/TAG & TTG/TAA & TTG/TAA & ATG/TAA & TTG/TAG & $\Pi T G / T A G$ & TTG/TAA & ATG/TAA \\
\hline $\operatorname{cox} 1$ & ATT/TAG & ATT/TAG & ATT/TAG & ATT/TAA & $\mathrm{GTT} / \mathrm{T}$ & $\mathrm{GTT} / \mathrm{T}$ & ATT/TAA & TTG/TAA & ATT/TAG \\
\hline ND6 & TAT/TAA & TAT/TAG & ATT/TAG & TGT/TAA & TAT/TAG & TAT/TAG & ATT/TAA & TTG/TAG & TTG/TAA \\
\hline СYTB & $\mathrm{ATT} / \mathrm{T}$ & $\mathrm{GTT} / \mathrm{T}$ & ATT/TAA & $\mathrm{ATT} / \mathrm{T}$ & $\mathrm{TCT} / \mathrm{T}$ & ATT/T & ATT/TAA & $\mathrm{ATT} / \mathrm{T}$ & $\mathrm{GTT} / \mathrm{T}$ \\
\hline $\operatorname{cox} 3$ & ATT/TAA & ATT/TAA & ATT/TAA & ATT/TAA & ATT/TAA & ATT/TAA & ATT/TAG & ATT/TAA & ATA/T \\
\hline NDL4 & GTA/TAA & GTA/TAA & TTG/TAA & GTA/TAA & $\mathrm{GTA} / \mathrm{T}$ & GTA/T & TTA/T & GTT/TAA & $\pi \mathrm{TG} / \mathrm{T}$ \\
\hline ND1 & $\mathrm{TTG} / \mathrm{T}$ & $\mathrm{TTG} / \mathrm{T}$ & $\mathrm{TTG} / \mathrm{T}$ & TTG/TAA & $\mathrm{TTG} / \mathrm{T}$ & $T \mathrm{TG} / \mathrm{T}$ & $\Pi \mathrm{TG} / \mathrm{T}$ & $T \mathrm{TG} / \mathrm{T}$ & TTG/TAA \\
\hline ATP6 & ATT/TAG & TTG/TAA & ATT/TAG & ATT/TAG & ATT/TAA & ATT/TAA & ATT/TAA & ATT/TAG & TTT/TAG \\
\hline $\cos 2$ & ATT/TAA & $\mathrm{ATT/T}$ & ATT/TA & ATT/TAA & ATT/TAA & ATT/TAA & ATT/TAA & ATT/TAA & ATT/TAG \\
\hline ND3 & CTT/TAG & $\mathrm{CTT} / \mathrm{T}$ & $\mathrm{CTT/TAG}$ & $\mathrm{CTT} / \mathrm{T}$ & $\mathrm{CTT} / \mathrm{T}$ & $\mathrm{CTT} / \mathrm{T}$ & $\mathrm{CCT} / \mathrm{T}$ & CTT/TAG & $\pi T / T$ \\
\hline ND5 & TाT/TAG & TTG/TAG & TTG/TAG & TाT/TAG & TाT/TAG & TाT/TAG & TाA/TAA & $\pi T / T$ & TाT/TAG \\
\hline
\end{tabular}

Information was taken from previous studies for B. malayi [24], D. immitis [23], O. volvulus [22], and S. digitata [25]. Information regarding the Wolbachia status of species from various subfamilies is reported in [2]. 


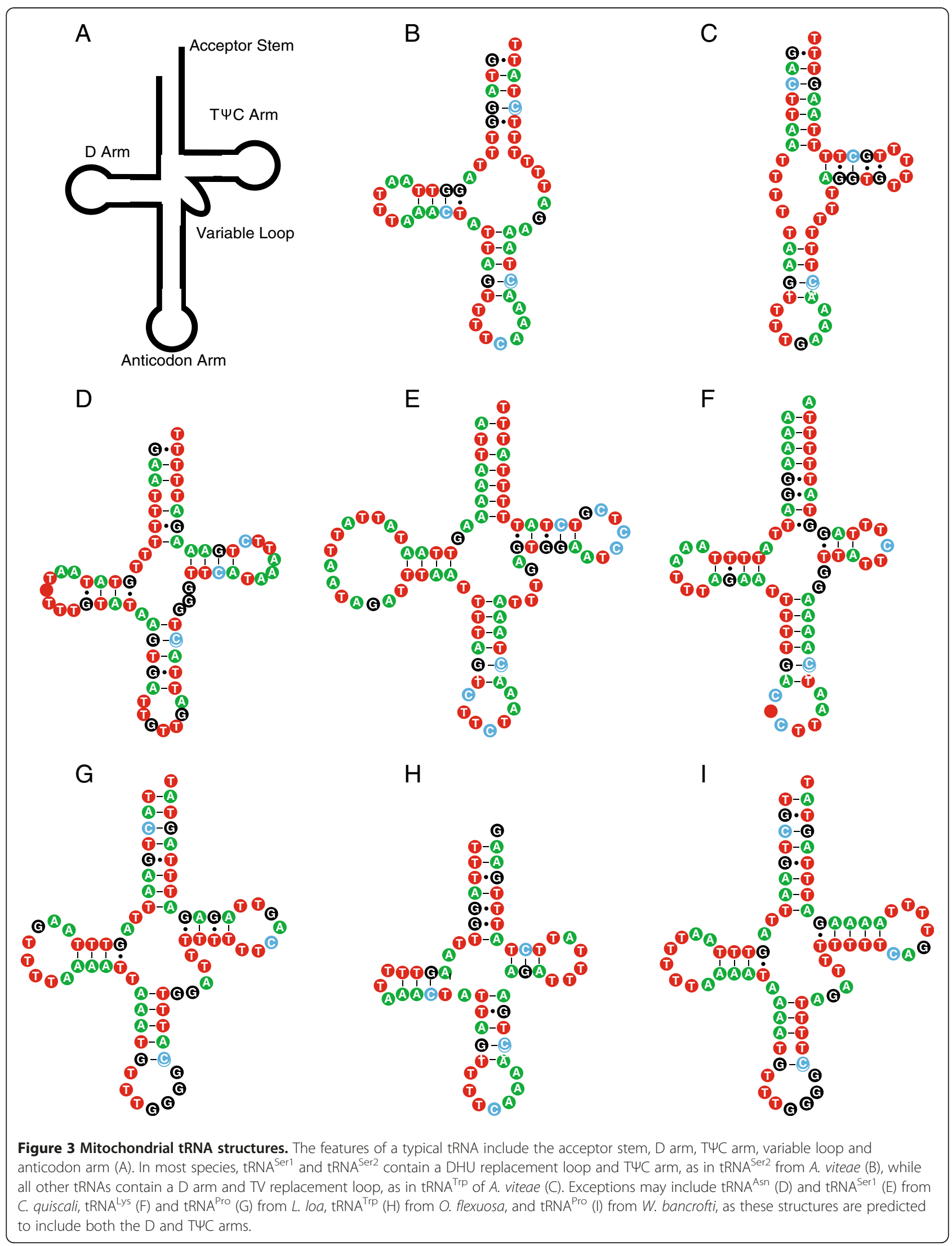


protein-coding, tRNA and rRNA genes. The AT rich regions of the 9 sequenced filarial mtDNAs range in size from $256 \mathrm{bp}$ in W. bancrofti to $506 \mathrm{bp}$ in S. digitata (Table 1). In most species, this region is located between Cox3 and tRNA ${ }^{\mathrm{Ala}}$. The unusual arrangement of tRNA genes in C. quiscali places its proposed 308 bp AT rich region between the tRNA ${ }^{\text {Met }}$ and $t R N A^{\text {Leu2 }}$ genes, leaving an additional 109 bp non-coding region between the Cox 3 and tRNA ${ }^{\text {Met }}$ genes. The function of this secondary non-coding region is unknown.

\section{Phylogenetic analysis}

A phylogenetic analysis was carried out using the nucleotide sequences of the 12 protein coding genes from the fully-sequenced filarial mtDNAs (Figure 4). Trees were left unrooted since the closest relatives of filarial nematodes with complete mtDNA sequences (i.e. Ascaris and Toxocara species) are still too divergent to allow for accurate alignment. Overall, topology is similar to that of trees based on single mitochondrial genes (i.e. 12s rRNA gene, Cox I) with improved statistical support [2,26,30]. As in the previous studies, our molecular phylogeny does not agree with the classical taxonomy of the filariae, as the Dirofilariinae and Onchocercinae appear as polyphyletic groups. In our tree, the lymphatic filariae cluster together, as do the genera Onchocerca and Dirofilaria. C. quiscali, which has not been included in previous analyses, is most closely related to the lymphatic filariae and $L$. loa. A. viteae and $S$. digitata are basal to the other species in our study.

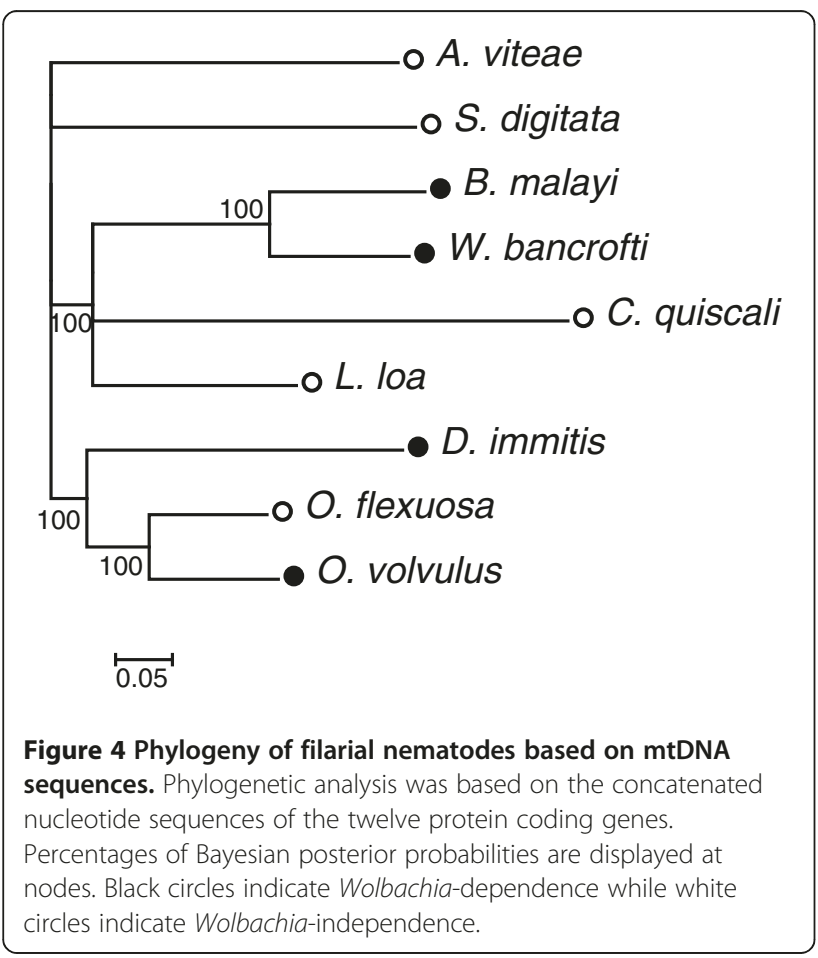

Simpler neighbor joining trees were constructed for each individual gene (data not shown). As in the tree based on concatenated protein coding sequences, clustering is never reflective of Wolbachia status.

\section{Discussion}

Filarial nematodes are widespread parasites that infect all classes of vertebrates except fish [31]. Many of these are of socioeconomic and medical importance. However, the mtDNAs had only been sequenced from 4 filarial species [22-25]. In our study we characterized the mtDNAs of 5 additional species and used the newly available sequences to compare the mtDNA of Wolbachia-dependent and independent filarial nematodes. Initially, we hypothesized that there might be obvious differences in the mitochondrial genome sequences of Wolbachia-dependent and independent species due to the evolutionary pressure exerted by a co-transmitted endosymbiont that may impact the energy balance of its host [14,16-21]. However, our data indicate that the mtDNAs of filarial nematodes are, thus far, remarkably similar. No major differences in genome length, AT content or codon usage were detected. All 9 species contain the standard 12 protein-coding genes, 22 tRNAs and 2 rRNAs. Differences in tRNA structure or anticodon usage are also minor and do not correlate to Wolbachia status. In light of these findings, it seems that Wolbachia has had little effect on the content of filarial mtDNA.

In most filarial species, synteny was perfectly preserved. The rearrangement of 5 tRNA's in C. quiscali probably reflects its evolutionary distance from the other species included in this study rather than its Wolbachiafree status (see Figure 4), as such rearrangements were not detected in other Wolbachia-free species. It will be necessary to sequence the mtDNAs of other members of the Splendidofilariinae in order to determine whether this rearrangement is species-specific or typical of the entire subfamily. However, this minor alteration in gene order is unlikely to impact overall mitochondrial function.

If Wolbachia infection had led to the fixation of certain mitochondrial types in an ancestral population, one might expect to see higher degrees of sequence identity between species that have come into contact with the endosymbiont (i.e. species from the Onchocercinae and Dirofilariinae) as compared to species that have not [2]. However, our phylogenetic analysis indicated that the level of sequence identity shared between mitochondrial protein-coding genes is independent of Wolbachia status. If it is true that filarial species considered primitive based on classical taxonomy (i.e. C. quiscali and S. digitata) were never associated with Wolbachia, the similarity of their mtDNAs to the others in this study makes it seem unlikely that Wolbachia have had a significant impact on mtDNA sequence. The lack of diversity in mtDNA sequences could 
be taken as an indication that the initial infection and theoretical Wolbachia-induced genetic bottleneck occurred in an ancestor of all filarial nematodes, but this is unlikely given the phylogenetic age of the filariae. Of course, one must consider that this study was performed on the level of complete genes. In the future, it may be informative to compare smaller loci in Wolbachia-dependent and independent species, as even single base changes are known to have profound effects on mitochondrial function [32].

The fact that no sequence-level differences were observed between mitochondria from Wolbachiadependent and independent filarial species does not exclude the possibility that differences may exist in mitochondrial function or efficiency between these groups. Of course, many of the genes related to oxidative phosphorylation and energy metabolism are encoded in the nuclear genome rather than the mitochondrial genome. Because only one filarial genome has been published (see [24] for the genome of B. malayi), we do not know if certain nuclear genes are missing or altered in Wolbachia-dependent species relative to their Wolbachia-independent counterparts.

Even if there are no differences in the genes encoded by Wolbachia-dependent and independent filarial worms, it is possible that variation in expression levels could lead to differences in mitochondrial output. Expression levels may be partially dictated by the number of mitochondrial genomes present in each mitochondria or the density of mitochondria in a given organism. These factors are also variable. Quantitative real-time PCR techniques could be used to assess expression; however, careful normalization would be necessary to ensure accurate results when comparing expression levels across multiple species and life cycle stages. This type of analysis will not be possible until better nuclear genome data is produced to provide appropriate control sequences.

Mitochondria and Wolbachia appear to share a common evolutionary story wherein bacteria of the Rickettsial family were taken up and transformed over time into an obligate mutualist that provides products essential to the life of the host. Since Wolbachia and mitochondria are co-transmitted and since Wolbachia may have an impact on host energy metabolism [14], it is possible that Wolbachia have affected the mitochondria of Wolbachia-dependent species in ways that are not reflected in the mtDNA sequence. Additional research will be required to test this hypothesis.

\section{Conclusions}

Here we report the mitochondrial genome sequences of 5 species of filarial nematodes: Acanthocheilonema viteae, Chandlerella quiscali, Loa loa, Onchocerca flexuosa, and Wuchereria bancrofti. Although we had hypothesized that the presence of Wolbachia endobacteria in some filarial nematodes may have had an impact on the content, organization or sequence of filarial mtDNA, we found no evidence that supports this hypothesis. The 9 available filarial mitochondrial sequences are remarkably similar on the sequence level. Future studies may determine whether functional differences exist between the mitochondria of Wolbachia-dependent and independent filarial nematodes.

\section{Methods}

\section{Parasite materials}

Adult B. malayi and A. viteae were obtained from experimentally infected Mongolian jirds as previously described [33,34]. Adult D. immitis were obtained from the Filariasis Research Reagent Resource Center (Athens, GA). Adult O. flexuosa were isolated from subcutaneous nodules dissected from red deer (Cervus elaphus) in Schleswig-Holstein, Germany [10]. Adult O. volvulus, and microfilariae of W. bancrofti and L. loa were available from prior studies in Uganda, Papua New Guinea and Cameroon, respectively [8,35,36]. Adult Chandlerella quiscali were obtained from common grackles Quiscalus quiscula trapped in North Dakota, USA.

\section{Nucleic acid isolation and cDNA synthesis}

DNA for sequencing was isolated from adult worms and microfilariae using the DNeasy Blood and Tissue Kit (Qiagen, Valencia, CA), ethanol precipitated to concentrate and stored in $1 \mathrm{x}$ TE buffer. RNA was isolated as previously described [29]. Briefly, worms were homogenized by bead-beating in TRIzol (Invitrogen, Carlsbad, CA) and RNA was isolated by organic extraction with 1-bromo-3chloropropane followed by column purification using the RNeasy Mini Kit (Qiagen) including an on-column DNase digest. A second DNase treatment was performed with the TURBO DNA-free Kit (Applied Biosystems, Austin, TX). cDNA was synthesized from total RNA using qScript cDNA SuperMix according to manufacturer's suggested protocol (Quant Biosciences, Gaithersburg, MD) and purified with the Qiaquick PCR Purification Kit (Qiagen).

\section{PCR reactions and sequencing}

Primers used to amplify mtDNA in 10 segments are reported in Additional file 2: Table S2. "Filarial Mito" primer sets are designed to target conserved portions of filarial mitochondria. In cases where the conserved primer set failed, species-specific primer sets were implemented. This was the case for segments $1-2,7$ and 9 in $A$. viteae, segments 5 and 8 in $C$. quiscali, segments $1,4,8$ and 10 in L. loa, and segments 7 and 9 in W. bancrofti. All PCR reactions were performed using the Platinum Taq High Fidelity DNA polymerase (Invitrogen) according to the manufacturer's suggested protocol with annealing temperatures adjusted to accommodate the thermodynamic properties of the primers. PCR products were cloned using the TOPO-TA Cloning Kit for Sequencing or the 
TOPO-XL PCR Cloning Kit (Invitrogen) depending on size, and sequenced by primer walking.

Species-specific primers (given "RT" designation in the primer name) were designed to detect protein-coding sequences from cDNA. The sequences of these primers are reported in Additional file 2: Table S2. To detect expression, each PCR reaction included a DNA positive control, a cDNA test sample and total RNA and wateronly negative controls.

\section{Assembly and annotation of the mitochondrial genomes}

Contigs were assembled using Contig Express and analyzed using Vector NTI version 10.3.1 (Invitrogen). Sequences were verified by comparison with publically available sequence data from the Genbank sequence read archive for L. loa (accession number SRP000756) and W. bancrofti (accession number SRP000772).

Protein-coding genes (including initiation and termination codons) and rRNAs were determined based on their homology to sequences reported from the mitochondrial genomes of $B$. malayi, D. immitis, O. volvulus and $S$. digitata [22-25].

In most instances, tRNA sequences were predicted using Arwen (available at http://130.235.46.10/ARWEN/) [37] and verified by homology to known filarial tRNA sequences. Any computationally predicted tRNAs that fell within other documented structures (i.e. protein-coding genes or rRNAs) were disregarded. tRNA ${ }^{\mathrm{Ala}}$ and tRNA ${ }^{\mathrm{Leu} 2}$ in A. viteae, tRNA ${ }^{\mathrm{Leu} 2}$ and tRNA ${ }^{\mathrm{Gly}}$ in O. flexuosa, and tRNA $^{\text {Ala }}$ in $L$. loa were identified solely based on homology to known orthologs and the presence of the expected anticodon.

Base composition and codon usage were calculated using the DNA Stats and codon usage features available from the Sequence Manipulation Suite (http://www.bioinformatics. org/sms2/dna_stats.html). Diagrams of complete mtDNAs were constructed using DNA plotter (http://www.sanger.ac. uk/resources/software/dnaplotter/) [38].

\section{Phylogenetic analysis}

The nucleotide sequences of the 12 protein coding genes, excluding stop codons, were aligned using Clustal $\mathrm{W}$ as implemented in MEGA4 using default parameters [39]. Individual gene alignments were concatenated using FASconCAT [40].

Model selection was performed using MrModeltest2.3 according to the Akaike information criterion [41]. Bayesian Metropolis-coupled Markov chain Monte Carlo (MCMC) analysis was performed on the dataset with the GTR + I + G nucleotide substitution model by MrBayes Version 3.1.2 [42,43]. Two simultaneous runs of 500,000 generations were performed with sampling every 100 generations and a $25 \%$ burn-in. The resulting phylogenetic tree was visualized in MEGA4 [39].

\section{Additional files} Additional file 1: Table S1. Codon usage in the mitochondrial
genomes of filarial nematodes

Additional file 2: Table S2. Primer sequences

\section{Abbreviations}

ATP6: ATP synthase subunit 6; CytB: Cytochrome b; Cox1: Cox2 and Cox3, cytochrome C oxidase subunits 1-3; ND1-6, and NDL4: Nicotinamide adenine dinucleotide dehydrogenase subunits 1-6, and L4; rRNA: Ribosomal RNA; tRNA: Transfer RNA.

\section{Competing interests}

The authors declare that they have no competing interests.

\section{Acknowledgements}

We would like to thank Richard Lucius (Humboldt University, Berlin), the Filariasis Research Reagent Resource Center (Atlanta, Georgia), Samuel Wanji (University of Buea, Cameroon), Norbert Brattig (Bernard Nocht Institute for Tropical Medicine, Hamburg) and Yuefang Huang (Washington University School of Medicine, St. Louis) for providing parasite material.

This research was funded by The Barnes Jewish Hospital Foundation. SNM was supported by NIH grant T32-Al007172 and JAV was supported by $\mathrm{NIH}$ grant R03-Al092306.

\section{Author details}

${ }^{1}$ Infectious Diseases Division, Department of Internal Medicine, Washington University School of Medicine, Campus Box 8051, 660 S. Euclid Avenue, St. Louis, MO 63110, USA. '2Department of Biology, University of North Dakota, 10 Cornell St, Grand Forks, ND 58202, USA.

\section{Authors' contributions}

SNM sequenced and assembled the mitochondrial genomes, carried out bioinformatic analyses and drafted the manuscript. ASM assisted in sequencing and assembling the mitochondrial genomes. JAV and WT collected parasite material and assisted in revising the manuscript. GJW and PUF supervised study design and assisted in drafting and revising the manuscript. All authors have read and approved the final manuscript.

Received: 9 November 2011 Accepted: 24 April 2012

Published: 24 April 2012

\section{References}

1. Bandi C, Anderson TJ, Genchi C, Blaxter ML: Phylogeny of Wolbachia in filarial nematodes. Proc Biol Sci 1998, 265(1413):2407-2413.

2. Ferri E, Bain O, Barbuto M, Martin C, Lo N, Uni S, Landmann F, Baccei SG, Guerrero R, de Souza Lima S, Bandi C, Wanji S, Diagne M, Casiraghi M: New Insights into the Evolution of Wolbachia Infections in Filarial Nematodes Inferred from a Large Range of Screened Species. PLoS One 2011, 6(6):e20843.

3. Kozek WJ, Marroquin HF: Intracytoplasmic bacteria in Onchocerca volvulus. AmJTrop Med Hyg 1977, 26(4):663-678.

4. Vincent $A L$, Ash LR, Frommes SP: The ultrastructure of adult Brugia malayi (Brug, 1927) (Nematoda: Filarioidea). J Parasitol 1975, 61(3):499-512.

5. Hoerauf A, Mand S, Fischer K, Kruppa T, Marfo-Debrekyei Y, Debrah AY, Pfarr KM, Adjei O, Buttner DW: Doxycycline as a novel strategy against bancroftian filariasis-depletion of Wolbachia endosymbionts from Wuchereria bancrofti and stop of microfilaria production. Med Microbiol Immunol 2003, 192(4):211-216.

6. Hoerauf A, Mand S, Volkmann L, Buttner M, Marfo-Debrekyei Y, Taylor M, Adjei O, Buttner DW: Doxycycline in the treatment of human onchocerciasis: Kinetics of Wolbachia endobacteria reduction and of inhibition of embryogenesis in female Onchocerca worms. Microbes Infect 2003, 5(4):261-273.

7. Hoerauf A, Nissen-Pahle K, Schmetz C, Henkle-Duhrsen K, Blaxter ML, Buttner DW, Gallin MY, Al-Qaoud KM, Lucius R, Fleischer B: Tetracycline therapy 
targets intracellular bacteria in the filarial nematode Litomosoides sigmodontis and results in filarial infertility. J Clin Invest 1999, 103(1):11-18.

8. Buttner DW, Wanji S, Bazzocchi C, Bain O, Fischer P: Obligatory symbiotic Wolbachia endobacteria are absent from Loa loa. Filaria J 2003, 2(1):10.

9. McNulty SN, Fischer K, Mehus JO, Vaughan JA, Tkach W, Weil GJ, Fischer PU: Absence of Wolbachia Endobacteria in Chandlerella quiscali, an Avian Filarial Parasite. J Parasitol 2012, 98(2):382-387.

10. Plenge-Bonig A, Kromer M, Buttner DW: Light and electron microscopy studies on Onchocerca jakutensis and O. flexuosa of red deer show different host-parasite interactions. Parasitol Res 1995, 81(1):66-73.

11. Andersson SG, Zomorodipour A, Andersson JO, Sicheritz-Ponten T, Alsmark UC, Podowski RM, Naslund AK, Eriksson AS, Winkler HH, Kurland CG: The genome sequence of Rickettsia prowazekii and the origin of mitochondria. Nature 1998, 396(6707):133-140.

12. Dumler JS, Barbet AF, Bekker CP, Dasch GA, Palmer GH, Ray SC, Rikihisa Y, Rurangirwa FR: Reorganization of genera in the families Rickettsiaceae and Anaplasmataceae in the order Rickettsiales: unification of some species of Ehrlichia with Anaplasma, Cowdria with Ehrlichia and Ehrlichia with Neorickettsia, descriptions of six new species combinations and designation of Ehrlichia equi and 'HGE agent' as subjective synonyms of Ehrlichia phagocytophila. Int J Syst Evol Microbiol 2001, 51(Pt 6):2145-2165.

13. Foster J, Ganatra M, Kamal I, Ware J, Makarova K, Ivanova N, Bhattacharyya A, Kapatral V, Kumar S, Posfai J, Vincze T, Ingram J, Moran L, Lapidus A, Omelchenko M, Kyrpides N, Ghedin E, Wang S, Goltsman E, Joukov V, Ostrovskaya O, Tsukerman K, Mazur M, Comb D, Koonin E, Slatko B: The Wolbachia genome of Brugia malayi: endosymbiont evolution within a human pathogenic nematode. PLOS Biol 2005, 3(4):e121

14. Strubing U, Lucius R, Hoerauf A, Pfarr KM: Mitochondrial genes for hemedependent respiratory chain complexes are up-regulated after depletion of Wolbachia from filarial nematodes. Int J Parasitol 2010, 40(10):1193-1202.

15. Werren JH, Baldo L, Clark ME: Wolbachia: master manipulators of invertebrate biology. Nat Rev Microbiol 2008, 6(10):741-751.

16. Delgado AM, Cook JM: Effects of a sex-ratio distorting endosymbiont on mtDNA variation in a global insect pest. BMC Evol Biol 2009, 9:49.

17. Hurst GD, Jiggins FM: Problems with mitochondrial DNA as a marker in population, phylogeographic and phylogenetic studies: the effects of inherited symbionts. Proc Biol Sci 2005, 272(1572):1525-1534.

18. Raychoudhury R, Grillenberger BK, Gadau J, Bijlsma R, van de Zande L, Werren JH, Beukeboom LW: Phylogeography of Nasonia vitripennis (Hymenoptera) indicates a mitochondrial-Wolbachia sweep in North America. Heredity 2010, 104(3):318-326

19. Turelli M, Hoffmann AA: Rapid spread of an inherited incompatibility factor in California Drosophila. Nature 1991, 353(6343):440-442.

20. Turelli M, Hoffmann AA, McKechnie SW: Dynamics of cytoplasmic incompatibility and mtDNA variation in natural Drosophila simulans populations. Genetics 1992, 132(3):713-723.

21. Belanger DH, Perkins SL: Wolbachia infection and mitochondrial diversity in the canine heartworm (Dirofilaria immitis). Mitochondrial DNA 2010, 21(6):227-233.

22. Keddie EM, Higazi T, Unnasch TR: The mitochondrial genome of Onchocerca volvulus: sequence, structure and phylogenetic analysis. $\mathrm{Mol}$ Biochem Parasitol 1998, 95(1):111-127.

23. Hu M, Gasser RB, Abs El-Osta YG, Chilton NB: Structure and organization of the mitochondrial genome of the canine heartworm, Dirofilaria immitis. Parasitology 2003, 127(Pt 1):37-51

24. Ghedin E, Wang S, Spiro D, Caler E, Zhao Q, Crabtree J, Allen JE, Delcher AL, Guiliano DB, Miranda-Saavedra D, Angiuoli SV, Creasy T, Amedeo P, Haas B, El-Sayed NM, Wortman JR, Feldblyum T, Tallon L, Schatz M, Shumway M, Koo H, Salzberg SL, Schobel S, Pertea M, Pop M, White O, Barton GJ, Carlow CK, Crawford MJ, Daub J, Dimmic MW, Estes CF, Foster JM, Ganatra M, Gregory WF, Johnson NM, Jin J, Komuniecki R, Korf I, Kumar S, Laney S, Li BW, Li W, Lindblom TH, Lustigman S, Ma D, Maina CV, Martin DM, McCarter JP, McReynolds L, Mitreva M, Nutman TB, Parkinson J, Peregrin-Alvarez JM, Poole C, Ren Q, Saunders L, Sluder AE, Smith K, Stanke M, Unnasch TR, Ware J, Wei AD, Weil G, Williams DJ, Zhang Y, Williams SA, Fraser-Liggett C, Slatko B, Blaxter ML, Scott AL: Draft genome of the filarial nematode parasite Brugia malayi. Science 2007, 317(5845):1756-1760.

25. Yatawara L, Wickramasinghe S, Rajapakse RP, Agatsuma T: The complete mitochondrial genome of Setaria digitata (Nematoda: Filarioidea): Mitochondrial gene content, arrangement and composition compared with other nematodes. Mol Biochem Parasitol 2010, 173(1):32-38.
26. Casiraghi M, Bain O, Guerrero R, Martin C, Pocacqua V, Gardner SL, Franceschi A, Bandi C: Mapping the presence of Wolbachia pipientis on the phylogeny of filarial nematodes: evidence for symbiont loss during evolution. Int J Parasitol 2004, 34(2):191-203.

27. McLaren DJ, Worms MJ, Laurence BR, Simpson MG: Micro-organisms in filarial larvae (Nematoda). Trans R Soc Trop Med Hyg 1975, 69(5-6):509-514.

28. McGarry HF, Pfarr K, Egerton G, Hoerauf A, Akue JP, Enyong P, Wanji S, Klager SL, Bianco AE, Beeching NJ, Taylor MJ: Evidence against Wolbachia symbiosis in Loa loa. Filaria J 2003, 2(1):9.

29. McNulty SN, Foster JM, Mitreva M, Dunning Hotopp JC, Martin J, Fischer K, Wu B, Davis PJ, Kumar S, Brattig NW, Slatko BE, Weil GJ, Fischer PU: Endosymbiont DNA in endobacteria-free filarial nematodes indicates ancient horizontal genetic transfer. PLoS One 2010, 5(6):e11029.

30. Casiraghi M, Anderson TJ, Bandi C, Bazzocchi C, Genchi C: A phylogenetic analysis of filarial nematodes: comparison with the phylogeny of Wolbachia endosymbionts. Parasitology 2001, 122(Pt 1):93-103.

31. Anderson RC and Bain O: Keys to genera of the order Spirurida. Part 3. Diplotriaenoidea, Aproctoidea and Filarioidea., in $\mathrm{ClH}$ keys to the nematode parasites of vertebrates, Anderson RC, Chabaud AG, and Willmott S, Editors. 1976, Commonwealth Agricultural Bureau: Farnham Royal, UK. p. 59-116.

32. Wong LJ: Molecular genetics of mitochondrial disorders. Dev Disabil Res Rev 2010, 16(2):154-162.

33. Ash LR, Riley JM: Development of subperiodic Brugia malayi in the jird, Meriones unguiculatus, with notes on infections in other rodents. $J$ Parasitol 1970, 56(5):969-973.

34. Lucius $\mathrm{R}$, Textor G: Acanthocheilonema viteae: rational design of the life cycle to increase production of parasite material using less experimental animals. Appl Parasitol 1995, 36(1):22-33.

35. Fischer P, Kipp W, Bamuhiga J, Binta-Kahwa J, Kiefer A, Buttner DW: Parasitological and clinical characterization of Simulium neavei-transmitted onchocerciasis in western Uganda. Trop Med Parasitol 1993, 44(4):311-321.

36. Weil GJ, Kastens W, Susapu M, Laney SJ, Williams SA, King CL, Kazura JW, Bockarie MJ: The impact of repeated rounds of mass drug administration with diethylcarbamazine plus albendazole on bancroftian filariasis in Papua New Guinea. PLoS Negl Trop Dis 2008, 2(12):e344.

37. Laslett D, Canback B: ARWEN: a program to detect tRNA genes in metazoan mitochondrial nucleotide sequences. Bioinformatics 2008, 24(2):172-175

38. Carver T, Thomson N, Bleasby A, Berriman M, Parkhill J: DNAPlotter: circular and linear interactive genome visualization. Bioinformatics 2009, 25(1):119-120.

39. Tamura K, Dudley J, Nei M, Kumar S: MEGA4: Molecular Evolutionary Genetics Analysis (MEGA) software version 4.0. Mol Biol Evol 2007, 24(8):1596-1599.

40. Kuck P, Meusemann K: FASconCAT: Convenient handling of data matrices. Mol Phylogenet Evol 2010, 56(3):1115-1118.

41. Nylander JAA, MrModeltest v2. 2004, Program distributed by the author, Evolutionary Biology Centre, Uppsala University

42. Huelsenbeck JP, Ronquist F: MRBAYES: Bayesian inference of phylogenetic trees. Bioinformatics 2001, 17(8):754-755.

43. Ronquist F, Huelsenbeck JP: MrBayes 3: Bayesian phylogenetic inference under mixed models. Bioinformatics 2003, 19(12):1572-1574.

doi:10.1186/1471-2164-13-145

Cite this article as: McNulty et al: Comparing the mitochondrial genomes of Wolbachia-dependent and independent filarial nematode species. BMC Genomics $201213: 145$.

\section{Submit your next manuscript to BioMed Central and take full advantage of:}

- Convenient online submission

- Thorough peer review

- No space constraints or color figure charges

- Immediate publication on acceptance

- Inclusion in PubMed, CAS, Scopus and Google Scholar

- Research which is freely available for redistribution 